\title{
El comercio de aves silvestres en la ciudad de Mérida, Yucatán, México
}

\author{
The wild bird trade in the city of Merida, Yucatan, Mexico \\ Linda Rosana González-Herrera ${ }^{1 *}$, Juan Chablé-Santos ${ }^{1}$, Wilian Aguilar-Cordero ${ }^{2}$, Pablo Manríque-Saide ${ }^{1}$ \\ ${ }^{1}$ Departamento de Zoología. Universidad Autónoma de Yucatán, Campus de Ciencias Biológicas y Agropecuarias. Carretera \\ Mérida-Xmatkuil Km. 15.5 Apartado postal, Itzimná, CP. 97100 Mérida, Yucatán, México. \\ ${ }^{2}$ Departamento de Botánica. Universidad Autónoma de Yucatán, Campus de Ciencias Biológicas y Agropecuarias. Carretera \\ Mérida-Xmatkuil Km. 15.5 Apartado postal, Itzimná, CP. 97100 Mérida, Yucatán, México. \\ *Autor de correspondencia: rosanagh9@gmail.com
}

Artículo científico recibido: 23 de octubre de 2016 aceptado: 09 de octubre de 2017

RESUMEN. El comercio de aves silvestres es una actividad que se practica desde tiempos prehispánicos y que persiste en la actualidad. Se ha sugerido que esta actividad es causa de disminución de poblaciones locales de algunas especies. El objetivo de este trabajo fue identificar las especies de aves silvestres que son comercializadas, y valorar la importancia de ellas dentro del comercio local, en mercados de la ciudad. Se realizaron observaciones directas en seis mercados, se aplicaron entrevistas a cinco comerciantes de aves y se aplicaron 125 cuestionarios a personas que acudieron a los mercados para adquirir aves. Se registraron 32 especies de aves pertenecientes a 13 familias y seis órdenes taxonómicos. Siete especies se encuentran en alguna categoría de riesgo de acuerdo con la Norma Mexicana. Las especies más abundantes son Spinus psaltria, Sporophila torqueola y Tiaris olivaceus. El cardenal (Cardinalis cardinalis) presentó el mayor IVU con 0.032 y el segundo valor más elevado de UST con $57.6 \%$. Esta especie es apreciada y demandada por su canto, color, como compañía y para capturar más aves. El comercio de aves silvestres es una actividad permanente y el gusto por adquirir aves silvestres como mascotas, es de amplia tradición.

Palabras clave: Aves, comercio, México, uso tradicional, valor de uso

ABSTRACT. The trade in wild birds is an activity that has been practiced since pre-Hispanic times and that still persists today. It has been suggested that this activity has resulted in a decrease in local populations of some species. The aim of this work was to identify the species of wild birds that are commercialized and assess their importance within local commerce in the city?s markets. Direct observations were made in six markets, interviews were conducted with five bird merchants and 125 questionnaires were applied to people who came to the markets to purchase birds. Thirty-two bird species belonging to 13 families and six taxonomic orders were recorded. Seven species are in some risk category according to the Mexican Standard. The most abundant species are Spinus psaltria, Sporophila torqueola and Tiaris olivaceus. The northern cardinal (Cardinalis cardinalis) had the highest IVU with 0.032 and the second highest UST value with $57.6 \%$. This species is appreciated and demanded for its song, color and as company, as well as to capture more birds. The trade in wild birds is a permanent activity and the taste for acquiring wild birds as pets has a long tradition.

Key words: Birds, trade, Mexico, traditional use, use value

\section{INTRODUCCIÓN}

Las aves son parte integral de la cultura mexicana, poseen gran importancia en las sociedades humanas, con usos y valores que varían con el tiempo, espacio y cultura. Se emplean de forma diversa, ya sea como alimento, ornato, amuletos, mascotas, medicina, en rituales, cetrería, arte plumario y recreación (Trejo 2006, Arévalo 2010). Mantener en cautiverio aves silvestres es una cos- 
tumbre que persiste en la población mexicana, lo que ha propiciado el comercio formal e informal (González et al. 2011), que en las últimas décadas se ha incrementado de una manera no regulada (Silva y Bernard 2012). Sólo en México se extrae cada año miles de individuos de aves silvestres de sus hábitats naturales para satisfacer la demanda que está en constante crecimiento (López e Îñigo 2009, SEMARNAT 2013).

La comercialización de aves, puede disminuir de manera contundente sus poblaciones y en algunos casos, conducirlas a su extinción (Guix et al. 1997). La comercialización de aves en Yucatán es de amplia tradición, pero esta actividad ocurre de forma irregular; por lo que no existe información oficial del número de individuos comercializado, ni de la temporalidad y frecuencia de las especies comercializadas. Por lo anterior el objetivo de este trabajo fue identificar las especies de aves silvestres que se comercializan, y su valor de importancia en el comercio local.

\section{MATERIALES Y MÉTODOS}

\section{Área de estudio}

La ciudad de Mérida, se localiza en la región noroccidental del estado de Yucatán, entre los $20^{\circ}$ $53^{\prime}$ y $21^{\circ} 03^{\prime} \mathrm{LN}$ y los $89^{\circ} 42^{\prime}$ y $89^{\circ} 33^{\prime} \mathrm{LO}$ (INEGI 2010). Los sitios de muestreo fueron seis mercados de la ciudad, tres establecidos y los tres de tipo ambulante. Los mercados establecidos cuentan con infraestructura para albergar a los comerciantes y operan todos los días de la semana, mientras que los mercados ambulantes se establecen un día a la semana y al término del día laboral, son retirados. Para guardar la identidad de los sitios se mantuvo el anonimato y se denominaron como ME1, ME2, ME3 (Mercados establecidos) y MA1, MA2, MA3 (Mercados ambulantes).

De junio de 2014 a enero de 2015, se realizaron observaciones directas en los mercados, un día al mes en los mercados ambulantes y dos días en los mercados establecidos, para verificar las especies en venta, el número de individuos por especie y los nombres locales. Para identificar las es- pecies se utilizaron las guías de campo de Sibley (2009), Howell y Webb (2010) y National Geographic (2010); la nomenclatura empleada fue la establecida por la Unión de Ornitólogos Americanos (AOU 2015). Se utilizaron como nombres comunes los de la región y cuando los informantes desconocían el nombre local, se recurrió al trabajo de Escalante et al. (2014). Los informantes clave se seleccionaron con base en: 1) que sean comerciantes de pájaros con más de 10 años de experiencia, 2) que tengan edades de entre 20 y 60 años y 3) que tuvieran disponibilidad al diálogo, a quienes les aplicaron entrevistas semiestructuradas. Para recabar información sobre preferencia y usos de las distintas especies se aplicaron 125 cuestionarios a personas que acudieron a los puntos de venta con la finalidad de adquirir un ave o a preguntar su precio.

Para obtener la abundancia relativa de las especies, se asignó abundancia del $100 \%$ a la especie con mayor número de individuos. La abundancia de las demás especies se calculó en relación con la especie de mayor abundancia. Para lo cual se dividió el total de observaciones de la especie i entre el total de observaciones de la especie más abundante y se multiplicó por 100. Con el porcentaje de abundancia obtenido de cada especie, se asignó las categorías de abundancia de Pettingill (1970): abundante (90 a $100 \%$ ), común (65 a 89\%), medianamente común (31 a $64 \%)$, no común $(10$ a $30 \%)$ y rara (1 a $9 \%)$.

La frecuencia relativa de aparición de las especies a lo largo del periodo de muestreo, se obtuvo dividiendo el número de visitas con presencia de la especie de interés entre el número total de visitas. Las categorías empleadas fueron: muy frecuente ( $71 \%$ o más), frecuente $(51 \%$ a $70 \%)$, poco frecuente $(21 \%$ a $50 \%)$ y esporádica (20\% o menos) (Chablé-Santos et al. 2008). Para obtener el uso significativo TRAMIL (UST) y el índice de valor de uso (IVU), se empleó el enfoque de consenso en las respuestas proporcionadas por los encuestados (Germosén 1995). El UST se calculó dividiendo el número de menciones de una especie, entre el número de informantes encuestados, con la fórmula: UST $=$ (número de menciones para cada especie / número de informantes encuestados) * 


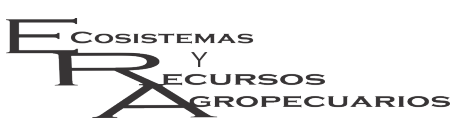

100. Para el IVU se utilizó la fórmula: IVU = número total de usos asignados a cada especie por los informantes / número de informantes encuestados. Se consideró un límite igual o superior a $20 \%$ para verificar la preferencia cultural de las especies. Estos valores expresan la importancia o valor cultural de una especie determinada para todos los informantes encuestados, como las especies más aceptadas y utilizadas (Toscano 2006, Pascual et al. 2014).

\section{RESULTADOS}

\section{Especies registradas y estatus de conservación}

Se registraron 32 especies de aves con uso comercial, las cuales pertenecen a 13 familias y seis órdenes taxonómicos. Las familias mejor representadas fueron Cardinalidae y Emberizidae con siete y cinco especies, respectivamente. Con respecto a su estatus de residencia fueron las especies residentes las mejor representadas con el $75 \%$ del total (Tabla 1). De acuerdo con las categorías de riesgo de las especies, se encontró que la Norma Mexicana incluye siete de ellas, tres amenazadas (Crax rubra (hocofaisán), Meleagris ocellata (pavo ocelado) y Ramphastos sulfuratus (tucán)), tres bajo protección especial (Eupsittula nana (kilí), Amazona albifrons (loro frente blanca) y Passerina ciris (mariposo)) y una en peligro de extinción Amazona oratrix (loro cabeza amarilla). Para la Unión Internacional para la Conservación de la Naturaleza, dos especies se encuentran bajo la categoría de casi amenazada ( $P$. ciris y $M$. ocellata); una como vulnerable ( $C$. rubra); una en peligro $(A$. oratrix) y 28 bajo la categoría de preocupación menor (Tabla 1 ).

\section{Especies y abundancias verificadas en los mer- cados de estudio}

En los seis mercados se observaron en venta 1 427 individuos de 23 especies; dos están en la categoría de abundantes: Spinus psaltria (chinchimbacal de capa) con 305 individuos y Sporophila torqueola (dominico) con 281, una especie es común, Tiaris olivaceus (tsilil) con 218 individuos y tres como medianamente comunes, Passerina cyanea (azulejo) Comercialización de aves en Mérida Ecosist. Recur. Agropec. 5(14):271-281,2018

con 176 individuos, Volatinia jacarina (huixito) con 107 y $P$. ciris con 94 . Mientras que 13 especies son raras, como Arremonops rufivirgatus (chocolatera) y las yuyas (Icterus spurius e I. gularis) (Tabla 2).

De acuerdo con la frecuencia de aparición de las especies en los mercados, nueve especies fueron muy frecuentes, cinco están presentes en todos los meses de muestreo (S. psaltria, P. cyanea, C. cardinalis, $T$. olivaceus y $S$. torqueola). En contraparte siete especies tienen frecuencia esporádica (Tabla 2). El mercado con mayor número de especies fue ME3 con 20, mientras que MA3 solo tuvo 6 especies. Las especies $S$. torqueola, S. psaltria, $P$. ciris, $T$. olivaceus y $V$. jacarina están presentes en todos los mercados (Tabla 2).

El componente de aves migratorias estuvo representado por ocho especies (Tabla 1), tuvo su mayor abundancia en los meses de noviembre, diciembre y enero, cuando las aves residentes presentan su menor abundancia (Figura 1). Las dos especies migratorias encargadas de estos cambios en la abundancia fueron $P$. cyanea y $P$. ciris, al constituir el $80 \%$ de la abundancia de las aves migratorias (Figura 2).

\section{Especies comercializadas según la percepción de los informantes clave}

De las entrevistas con pajareros, se obtuvo información de 17 especies que son comercializadas. Las especies que más se venden son $C$. cardinalis, $P$. ciris, P. cyanea, S. torqueola y $S$. psaltria. Apareciendo cuatro especies no verificadas en los mercados, las cuales son: Ortalis vetula (chachalaca), C. rubra (hocofaisán), M. ocellata y Cyanocorax yucatanicus (chel) (Tabla 1). Los informantes clave indican que son los pájaros machos los más demandados por los compradores, preferencia que se debe a su canto y color. Las personas que más adquieren aves son los hombres y solo un entrevistado mencionó que tanto hombres como mujeres compran por igual, realizándose la actividad de comercialización de aves silvestres todo el año. Teniendo los meses de noviembre a febrero la mayor demanda o compra de aves. 
González-Herrera et al.

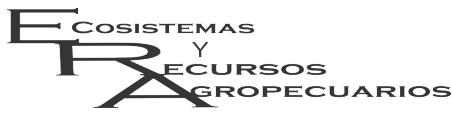

Comercialización de aves en Mérida

Ecosist. Recur. Agropec.

5(14):271-281,2018

Tabla 1. Especies de aves silvestres registradas en los mercados de la ciudad de Mérida, Yucatán.

\begin{tabular}{|c|c|c|c|c|c|c|c|}
\hline \multirow[t]{2}{*}{ Especie } & \multirow[t]{2}{*}{ Nombre común } & \multicolumn{3}{|c|}{ Técnicas } & \multirow[t]{2}{*}{ NOM } & \multirow[t]{2}{*}{ Estatus } & \multirow[t]{2}{*}{$\mathrm{UICN}$} \\
\hline & & OD & $\mathrm{C}$ & $\mathrm{E}$ & & & \\
\hline \multicolumn{8}{|l|}{ Orden Anseriformes } \\
\hline \multicolumn{8}{|l|}{ Familia Anatidae } \\
\hline Dendrocygna autumnalis (Linnaeus, 1758) & Pijijí & $\mathrm{X}$ & & & & $\mathrm{R}$ & LC \\
\hline \multicolumn{8}{|l|}{ Orden Galliformes } \\
\hline \multicolumn{8}{|l|}{ Familia Cracidae } \\
\hline Ortalis vetula (Wagler, 1830) & Chachalaca & & & $\mathrm{X}$ & & $\mathrm{R}$ & LC \\
\hline Crax rubra (Linnaeus, 1758) & Hocofaisán & & & $x$ & A & $\mathrm{R}$ & VU \\
\hline Meleagris ocellata (Cuvier, 1820) & Pavo ocelado & & & $x$ & $A, e$ & $\mathrm{R}$ & NT \\
\hline \multicolumn{8}{|l|}{ Orden Columbiformes } \\
\hline \multicolumn{8}{|l|}{ Familia Columbidae } \\
\hline Columbina talpacoti (Temminck, 1811) & Tortolita & & $x$ & & & $\mathrm{R}$ & LC \\
\hline Zenaida asiatica (Linnaeus, 1758) & Sacpakal & $\mathrm{X}$ & $\mathrm{X}$ & & & $\mathrm{R}$ & LC \\
\hline Leptotila verreauxi (Bonaparte, 1855) & Dzudzuy & & $x$ & & & $\mathrm{R}$ & LC \\
\hline \multicolumn{8}{|l|}{ Orden Piciformes } \\
\hline \multicolumn{8}{|l|}{ Familia Ramphastidae } \\
\hline Ramphastos sulfuratus (Lesson, 1830) & Tucán pico canoa & & $\mathrm{x}$ & & A & $\mathrm{R}$ & LC \\
\hline \multicolumn{8}{|l|}{ Orden Psittaciformes } \\
\hline \multicolumn{8}{|l|}{ Familia Psittacidae } \\
\hline Eupsittula nana (Vigors, 1830) & Kilí & $\mathrm{X}$ & $\mathrm{X}$ & $\mathrm{x}$ & $\operatorname{Pr}$ & $\mathrm{R}$ & LC \\
\hline Amazona albifrons (Sparrman, 1788) & Loro frente blanca & $\mathrm{X}$ & $\mathrm{X}$ & & $\operatorname{Pr}$ & $\mathrm{R}$ & LC \\
\hline Amazona oratrix (Ridgway, 1887) & Loro cabeza amarilla & & $\mathrm{x}$ & $x$ & $\mathrm{P}$ & $\mathrm{R}$ & EN \\
\hline \multicolumn{8}{|l|}{ Orden Passeriformes } \\
\hline \multicolumn{8}{|l|}{ Familia Corvidae } \\
\hline Cyanocorax yucatanicus (Dubois, 1875) & Chara yucateca & & & $\mathrm{x}$ & e & $\mathrm{R}$ & LC \\
\hline \multicolumn{8}{|l|}{ Familia Turdidae } \\
\hline Turdus grayi (Bonaparte, 1838) & Xcokita & $\mathrm{X}$ & $\mathrm{X}$ & & & $\mathrm{R}$ & LC \\
\hline Familia Mimidae & & & & & & & \\
\hline Dumetella carolinensis (Linnaeus, 1766) & Clarín & $\mathrm{x}$ & $\mathrm{X}$ & & & $\mathrm{M}$ & LC \\
\hline Mimus gilvus (Vieillot, 1808) & Centzontle & & $x$ & & & $\mathrm{R}$ & LC \\
\hline Volatinia jacarina (Linnaeus, 1766) & Huixito & $\mathrm{X}$ & $\mathrm{X}$ & $\mathrm{x}$ & & $\mathrm{R}$ & LC \\
\hline Sporophila torqueola (Bonaparte, 1850) & Dominico & $\mathrm{x}$ & $\mathrm{x}$ & $\mathrm{x}$ & & $\mathrm{R}$ & LC \\
\hline Tiaris olivaceus (Linnaeus, 1766) & Tsilil & $\mathrm{x}$ & $x$ & $\mathrm{x}$ & & $\mathrm{R}$ & LC \\
\hline Arremonops rufivirgatus (Lawrence, 1851) & Chocolatera & $\mathrm{X}$ & $\mathrm{X}$ & $\mathrm{x}$ & & $\mathrm{R}$ & LC \\
\hline Spizella pallida (Swainson, 1832) & Gorrión pálido & $\mathrm{x}$ & $\mathrm{X}$ & & & $\mathrm{M}$ & LC \\
\hline Familia Cardinalidae & & & & & & & \\
\hline Piranga olivacea (Linnaeus, 1758) & Colegial & $x$ & & & & M & LC \\
\hline Cardinalis cardinalis (Linnaeus, 1758) & Cardenal & $x$ & $x$ & $x$ & & $\mathrm{R}$ & LC \\
\hline Pheucticus ludovicianus (Linnaeus, 1766) & Degollado & $x$ & $x$ & $x$ & & M & LC \\
\hline Cyanocompsa parellina (Bonaparte, 1850) & Azulejo rey & $\mathrm{X}$ & $\mathrm{x}$ & $x$ & & $\mathrm{R}$ & LC \\
\hline Passerina caerulea (Linnaeus, 1758) & Azulejo chino & $\mathrm{X}$ & $\mathrm{X}$ & $\mathrm{x}$ & & $\mathrm{M}$ & LC \\
\hline Passerina cyanea (Linnaeus, 1766) & Azulejo & $\mathrm{X}$ & $\mathrm{X}$ & $\mathrm{x}$ & & $\mathrm{M}$ & LC \\
\hline Passerina ciris (Linnaeus, 1758) & Mariposo & $\mathrm{X}$ & $\mathrm{X}$ & $\mathrm{x}$ & $\operatorname{Pr}$ & $\mathrm{M}$ & NT \\
\hline Familia Icteridae & & & & & & & \\
\hline Icterus spurius (Linnaeus, 1758) & Yuya & $\mathrm{X}$ & $\mathrm{X}$ & & & $\mathrm{M}$ & LC \\
\hline Icterus gularis (Wagler, 1829) & Yuya & $\mathrm{X}$ & $\mathrm{X}$ & & & $\mathrm{R}$ & LC \\
\hline Familia Fringillidae & & & & & & & \\
\hline Euphonia affinis (Lesson, 1842) & Chinchimbacal platanero & $\mathrm{X}$ & $\mathrm{X}$ & & & $\mathrm{R}$ & LC \\
\hline Spinus psaltria (Say, 1823) & Chinchimbacal de capa & $\mathrm{X}$ & $\mathrm{X}$ & $\mathrm{x}$ & & $\mathrm{R}$ & LC \\
\hline Familia Estrildidae & & & & & & & \\
\hline Lonchura malaca (Linnaues, 1766) & Capuchino & $\mathrm{x}$ & & & & $\mathrm{R}$ & LC \\
\hline
\end{tabular}


González-Herrera et al.

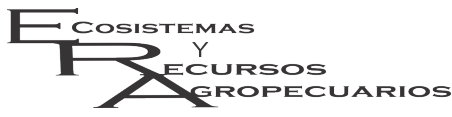

Comercialización de aves en Mérida

Ecosist. Recur. Agropec.

5(14):271-281,2018

Tabla 2. Abundancia relativa y frecuencia de aparición de las especies de aves registradas en los mercados de ciudad de Mérida, Yucatán.

\begin{tabular}{|c|c|c|c|c|c|c|c|c|c|}
\hline \multirow[t]{2}{*}{ Especie } & \multicolumn{9}{|c|}{ Mercados } \\
\hline & ME1 & ME2 & ME3 & MA1 & MA2 & MA3 & Total & $\mathrm{AR}$ & FA \\
\hline Dendrocygna autumnalis & & & 1 & & & & 1 & $\mathrm{R}$ & $E$ \\
\hline Zenaida asiatica & & & 4 & & & & 4 & $\mathrm{R}$ & $\mathrm{PF}$ \\
\hline Eupsittula nana & 46 & 1 & 1 & & & & 48 & $N C$ & MF \\
\hline Amazona albifrons & 8 & 6 & 16 & 2 & & 1 & 33 & NC & $\mathrm{F}$ \\
\hline Turdus grayi & & & 1 & & & & 1 & $\mathrm{R}$ & $\mathrm{E}$ \\
\hline Dumetella carolinensis & & & 1 & 1 & & & 2 & $\mathrm{R}$ & $\mathrm{PF}$ \\
\hline Volatinia jacarina & 16 & 20 & 53 & 9 & 6 & 3 & 107 & $\mathrm{MC}$ & MF \\
\hline Sporophila torqueola & 25 & 66 & 116 & 36 & 20 & 18 & 281 & A & MF \\
\hline Tiaris olivaceus & 50 & 47 & 84 & 22 & 11 & 4 & 218 & C & MF \\
\hline Arremonops rufivirgatus & 1 & 2 & 1 & 1 & & & 5 & $\mathrm{R}$ & $\mathrm{PF}$ \\
\hline Spizella pallida & & & 1 & & & & 1 & $\mathrm{R}$ & $\mathrm{E}$ \\
\hline Piranga rubra & & & 1 & & & & 1 & $\mathrm{R}$ & $\mathrm{E}$ \\
\hline Cardinalis cardinalis & 6 & 22 & 12 & 17 & & & 57 & $\mathrm{NC}$ & MF \\
\hline Pheucticus ludovicianus & 19 & 9 & 15 & 7 & 2 & & 52 & $\mathrm{NC}$ & MF \\
\hline Cyanocompsa parellina & 7 & & & & & & 7 & $\mathrm{R}$ & $\mathrm{E}$ \\
\hline Passerina caerulea & 2 & & 8 & & & & 10 & $\mathrm{R}$ & $\mathrm{F}$ \\
\hline Passerina cyanea & 59 & 27 & 66 & 23 & 1 & & 176 & $M C$ & MF \\
\hline Passerina ciris & 9 & 22 & 51 & 6 & 1 & 5 & 94 & $\mathrm{MC}$ & MF \\
\hline Icterus spurius & & & 1 & & & & 1 & $\mathrm{R}$ & $\mathrm{E}$ \\
\hline Icterus gularis & & & & 1 & & & 1 & $\mathrm{R}$ & $\mathrm{E}$ \\
\hline Euphonia affinis & 10 & & & & & & 10 & $\mathrm{R}$ & $\mathrm{PF}$ \\
\hline Spinus psaltria & 48 & 52 & 168 & 29 & 5 & 3 & 305 & A & MF \\
\hline Lonchura malaca & 5 & 2 & 2 & & 3 & & 12 & $\mathrm{R}$ & $\mathrm{PF}$ \\
\hline
\end{tabular}

Mercados de estudio: ME1 = Mercado Establecido 1, ME2 = Mercado Establecido 2, ME3 = Mercado Establecido 3, MA1 = Mercado Ambulante 1, MA2 = Mercado Ambulante 2, MA3 = Mercado Ambulante 3. Abundancia relativa ( $A R$ ): $A=A$ bundante, $C=$ Común, $M C=$ Medianamente común, $\mathrm{NC}=$ No común, $\mathrm{R}=$ Rara. Frecuencia relativa $(\mathrm{FR}): \mathrm{MF}=$ Muy frecuente, $\mathrm{F}=$ Frecuente, $\mathrm{PF}=$ Poco frecuente, $\mathrm{E}=$ Esporádica.

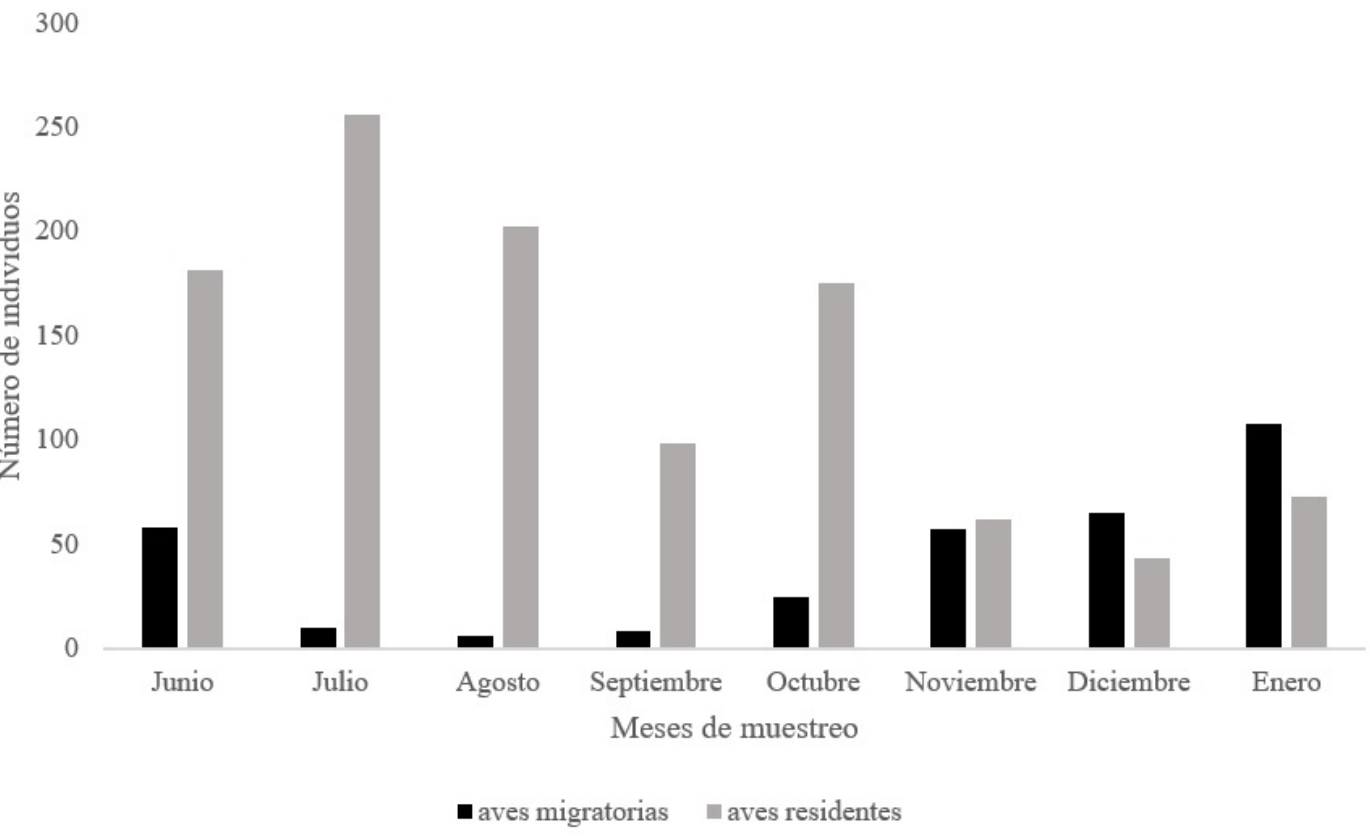

Figura 1. Abundancia de aves residentes y migratorias por mes de estudio. 


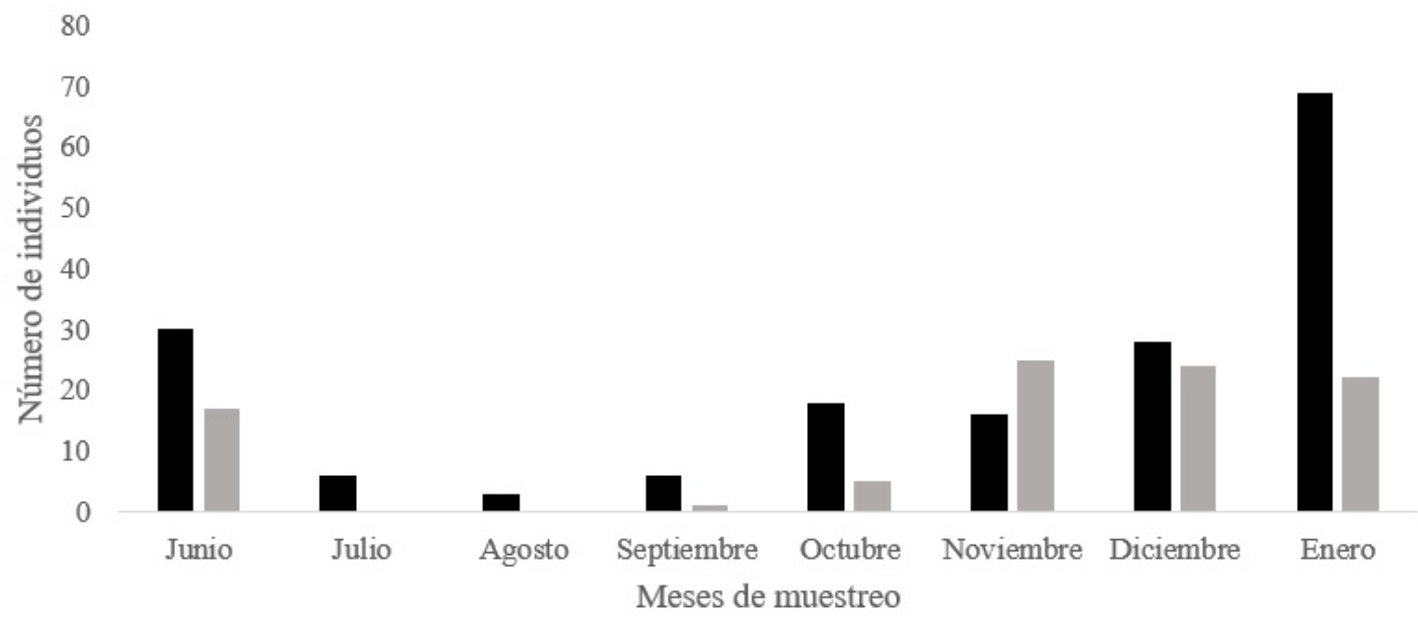

- Passerina cyanea $\quad$ Passerina ciris

Figura 2. Abundancia mensual de Passerina cyanea y Passerina ciris en los seis mercados de estudio.

Tabla 3.Uso Significativo TRAMIL (UST), Índice de Valor de Uso (IVU) y usos reportados de aves silvestres comercializadas en la ciudad de Mérida, Yucatán.

\begin{tabular}{lccc}
\hline Especie & UST (\%) & IVU & Usos \\
\hline Cardinalis cardinalis & 57.6 & 0.032 & Canto, color, compañía, "pescar" \\
Passerina cyanea & 61.6 & 0.024 & Canto, color, compañía \\
Spinus psaltria & 40.8 & & \\
Passerina ciris & 39.2 & & \\
Amazona albifrons & 22.4 & & \\
Sporophila torqueola & 30.4 & & \\
Tiaris olivaceus & 22.4 & & \\
Eupsittula nana & 15.2 & & \\
Volatinia jacarina & 8 & & \\
Cyanocompsa parellina & 6.4 & & Color, comestible, compañía \\
Zenaida asiatica & 4 & & Canto, compañía \\
Columbina talpacoti & 1.6 & & \\
Amazona oratrix & 1.6 & 0.016 & Canto, color \\
Turdus grayi & 1.6 & & \\
Pheucticus ludovicianus & 18.4 & & Color \\
Passerina caerulea & 11.2 & & \\
Arremonops rufivirgatus & 0.8 & 0.008 & \\
Mimus gilvus & 0.8 & & \\
Leptotila verreauxi & 0.8 & & \\
Dumetella carolinensis & 0.8 & & \\
Ramphastos sulfuratus & 0.8 & & \\
Spizella pallida & 0.8 & & \\
Icterus spurius & 0.8 & & \\
Icterus gularis & 0.8 & & \\
Euphonia affinis & 0.8 & & \\
\hline
\end{tabular}

Índice de valor de uso (IVU) y de uso significativo TRAMIL (UST)

Se encuestaron hombres de entre 12 y 74 años de edad (54.4\%) y mujeres de entre 11 y 80 años (45.6\%), quienes reportaron un listado de 25 especies de aves silvestres comercializadas (Tabla 1). Siendo los usos de canto, color, compañía, para pescar (capturar) y comestible los que motivan la 
compra de aves (Tabla 3). EI IVU de las especies comercializadas, señala al cardenal ( $C$. cardinalis) como el de mayor valor (0.032), el cual es adquirido por su canto, color, compañía y como señuelo para capturar otras aves. Diez especies tuvieron un IVU intermedio (0.024), entre las que se encuentran $E$. nana, S. torqueola, $P$. ciris y $C$. talpacoti, esta última se adquiere para consumo alimenticio. Con respecto al UST, siete especies tienen valores superiores al $20 \%$, siendo las especies con mayor valor P. cyanea $(61.6 \%)$ y C. cardinalis $(57.6 \%)$. Mientras que cinco especies tienen valores intermedios de entre 20 y $40 \%$ ( $A$. albifrons, S. torqueola, $T$. olivaceus, $P$. ciris y $S$. psaltria) todas adquiridas por canto, color y como compañía (Tabla 3).

\section{DISCUSIÓN}

En el estado de Yucatán se encuentran 456 especies de aves (Chablé-Santos y Pasos-Enríquez 2010), de las cuales el $7 \%$ se comercializa en los mercados. De las especies comercializadas 15 se encuentran en la guía de aves canoras, que hasta 2001 se encontraban permitidas para su captura (SEMARNAT 2001). Actualmente, en el estado no se tienen calendarios, ni listas oficiales para aprovechamiento de aves canoras y de ornato. Tras la aparición de las unidades de manejo y aprovechamiento sustentable de vida silvestre (UMA's), cualquier especie de vida silvestre de flora y fauna puede ser aprovechada bajo el esquema de UMA (SEMARNAP 1997).

De las especies comercializadas, los Passeriformes fueron los mejor representados con 21 especies, seguido de los Columbiformes y Psittaciformes, lo que coincide con otros trabajos (Aguilar 1992, Gómez et al. 2005, Trejo 2006). Características propias de este grupo son el plumaje colorido y la emisión de cantos elaborados, lo que influye en la demanda por parte de los compradores. Las aves residentes fueron las mejor representadas, en abundancia y frecuencia de aparición en la mayoría de los mercados. Destacando S. psaltria como la especie más abundante, la cual es de amplia distribución en el país (Howell y Webb 2010). En la región, esta especie se observa en zonas abiertas de selva baja hasta zonas urbanas, en especial dentro de la ciudad de Mérida, lo que facilita su captura. Otra especie frecuente fue $P$. cyanea, ave migratoria que llega a la zona a finales de octubre y se les puede observar hasta abril en grandes parvadas, principalmente en zonas abiertas y ambientes perturbados con arbustos y pastos en donde encuentra disponibilidad de semillas para alimentarse (Chablé-Santos et al. 2007). La temporada migratoria de esta especie concuerda con su presencia en los distintos mercados, donde se observa un incremento de individuos en estos meses. El componente migratorio, presentó su mayor abundancia en los meses de noviembre, diciembre y enero, para $P$. ludovicianus, $P$. cyanea y $P$. ciris. Es en estos meses cuando estas especies se encuentran haciendo uso de los ambientes presentes en la Península de Yucatán, permaneciendo de seis a siete meses en la zona para luego retornar a finales de febrero y marzo, a sus zonas de reproducción (Kaufman 2005, National Geographic 2010). Con respecto al UST se encontró que $P$. cyanea fue la especie con mayor valor $(61.6 \%)$ junto con $C$. cardinalis $(57.6 \%)$, resaltando su aceptación cultural por parte de la población que utiliza estas aves como ornato. El mariposo ( $P$. ciris) con un UST de 39.2 se encuentra bajo protección especial (DOF 2010), lo que no evita que se comercialice en los seis mercados, realizándose su exhibición y venta sin restricción. Los individuos comercializados, van desde etapas juveniles y hembras con su plumaje verde, llamados coloquialmente verdines, hasta machos adultos.

De especial preocupación fueron los psitácidos ( $A$. albifrons y $E$. nana). En el 2008 se realizó una modificación a la Ley General de Vida Silvestre (SEMARNAT 2008), estableciéndose que ningún psitácido mexicano puede ser sujeto de aprovechamiento extractivo con fines de subsistencia o comerciales. Lo que no evita la comercialización de $A$. albifrons en la mayoría de los mercados, además es una de las siete especies con mayor UST y mayor abundancia en los meses de mayo y junio. Esto parece deberse a que la temporada reproductiva de esta especie en la región se 
presenta principalmente entre abril y junio (ChabléSantos et al. 2007, Howell y Webb 2010), por lo que los individuos en venta son polluelos de apenas días de nacidos que son extraídos de los nidos para venta en los mercados.

De las entrevistas con informantes clave, se obtuvo que las especies $O$. vetula, $C$. rubra, $M$. ocellata y $C$. yucatanicus, son poco ofertadas, lo que ocasionó que no fueran registradas en las observaciones de los mercados. De acuerdo con los informantes, su ausencia se debe a que son aves de talla mediana a grande, lo que dificulta su traslado hasta la zona de venta, además de que requieren más alimento y alojamiento de mayores dimensiones. Una especie registrada por entrevista y encuesta, pero sin registro en los mercados fue $A$. oratrix, la cual se encuentra en peligro de extinción (DOF 2010, CITES 2011, UICN 2012). Esta especie ha disminuido su venta debido a su restricción, además de los precios elevados.

Los informantes, coinciden en señalar a $C$. cardinalis, $P$. ciris, $P$. cyanea, $S$. torqueola y $S$. psaltria como las especies más comercializadas, con preferencia por los machos. Tres de estas son residentes permanentes en la zona, pudiendo ser capturadas todo el año, siendo los machos los más apreciados por sus colores llamativos y cantos elaborados. Lo que también ha sido reportado por Drews (2002) y Acosta (2013) quienes señalan que las aves suelen ser atractivas por su canto, plumaje, porque brindan compañía o estatus a sus dueños, y para uso comestible. De acuerdo con el IVU, $C$. cardinalis es la especie con mayor valor, al ser adquirida por su canto, color, compañía, y por la característica exclusiva de esta especie, que se utiliza para atraer y capturar otras aves canoras territoriales (Aguilar 1992, Uc y Cervera 2014). Estudios realizados por Gómez et al. (2005) en tianguis y mercados también reportan al cardenal como la especie con mayor demanda, por su canto, colorido, y por su fácil adaptación al cautiverio. Al respecto Aguilar (1992) la reporta como la más comercializada y cotizada. Lo que coincide con su abundancia encontrada en los mercados y su alto UST.
Con respecto a los valores IVU, los psitácidos son las aves preferidas, a pesar de su elevado precio de venta. Al respecto Engebretson (2006) indica que la preferencia como ave de compañía puede atribuirse a su alto grado de imitación de sonidos. También se observaron aves de plumaje poco llamativo como $T$. grayi, $C$. talpacoti y $M$. gilvus con bajos IVU. En estos casos su captura suele ser accidental, al caer en las trampas colocadas con el objetivo de atrapar a otras especies de mayor interés (Acosta 2013). Sobre esto Bertonatti (1995) señala que el comercio de aves silvestres significa para algunos sectores de menores recursos un medio de subsistencia, pero para cumplir este objetivo, su aprovechamiento debe realizarse de modo sustentable. Es difícil valorar el impacto que la comercialización ejerce sobre las poblaciones locales de aves silvestres. Por trabajos realizados en psitácidos y otras aves canoras (Cantú et al. 2007, Monterrubio 2007, Escalona y Plasencia 2012), se sugiere que la excesiva captura constituye una fuerte limitante poblacional para estas especies (Ramírez 2010). Este trabajo es una aproximación al proceso de comercialización de aves silvestres en la ciudad de Mérida, Yucatán y aporta información de las especies utilizadas de forma tradicional como aves canoras y de ornato, lo que ofrece bases para el desarrollo de estrategias para la conservación y manejo.

\section{CONCLUSIONES}

El comercio de aves silvestres en los mercados de la ciudad de Mérida es una actividad permanente, y el gusto por adquirir aves silvestres como mascotas, es de amplia tradición. Las especies $S$. psaltria, $S$. torqueola, $T$. olivaceus, $P$. cyanea y $V$. jacarina fueron las más abundantes y frecuentes en los mercados. También fueron junto con $C$. cardinalis, $P$. ciris y $A$. albifrons las de mayor valor de uso y uso significativo. El aprovechamiento de estas especies requiere de acciones encaminadas en el corto y mediano plazo al manejo sustentable. 


\section{LITERATURA CITADA}

Acosta M (2013) Caza y comercio ilegal de aves silvestres en la provincia de Santa Fe, Argentina. BioScriba 6: 09-15.

Aguilar R (1992) El comercio de aves silvestres vivas en la ciudad de Xalapa Veracruz. Revista la Ciencia y el Hombre 11: 47-60.

Arévalo E (2010) Evaluación de las aves silvestres mantenidas en cautiverio en comunidades cercanas al Volcán Póas, Costa Rica. Asociación Ornitológica de Costa Rica. Zeledonia 14: 2-11.

AOU (2014) Checklist of North and Middle American Birds. American Ornithologists' Union. http://checklist. aou.org/taxa/2014. Fecha de consulta: 24 de abril de 2015.

Bertonatti C (1995) Se puede conservar aves silvestres ... ¿vendiéndolas? Nuestras aves, Revista de la Asociación Ornitológica del Plata 13: 7-9.

Cantú J, Sánchez M, Grosselete M, Silva, J (2007) Tráfico ilegal de pericos en México. Una evaluación detallada. Defenders of Wildlife. México. http://www.defenders.org/publications/trafico_ilegal_de _pericos_en_mexico.pdf. Fecha de consulta: 25 de abril de 2015.

Chablé-Santos J, Gómez-Uc E, Pasos-Enríquez R (2007) Aves comunes del Sur de Yucatán. Universidad Autónoma de Yucatán. Yucatán, México. 137p.

Chablé-Santos J, González-Rojas JI, Peña-Peniche A, Pasos-Enríquez R (2008) Bird diversity in the petenes of Yucatan State, Mexico. Ornitología Neotropical 19: 55-70.

Chablé-Santos J, Pasos-Enríquez R (2010) Aves. En: Durán R, Méndez M (eds.) Biodiversidad y Desarrollo Humano en Yucatán, CICY., PPD-FMAM, CONABIO, SEDUMA, UADY. Yucatán, México. pp: 264266.

CITES (2011) Especies CITES. Convención sobre el Comercio Internacional de Especies Amenazadas de Fauna y Flora Silvestres. http://www.cites.org/esp/disc/species.php. Fecha de consulta: 25 de abril de 2015.

DOF (2010) Diario Oficial de la Federación. Norma Oficial Mexicana NOM-059-SEMARNAT-2010. Protección ambiental-Especies nativas de México de flora y fauna silvestres-Categorías de riesgo y especificaciones para su inclusión, exclusión o cambio-Lista de especies en riesgo. 30 de diciembre de 2010, México. $78 p$.

Drews C (2002) Mascotas silvestres en hogares ticos: percepciones, actitudes y conocimientos. Ambientico 103: $12-13$.

Engebretson M (2006) The Welfare and Suitability of Parrots as Companion Animals: A Review. Animal Welfare 15: 263-276.

Escalante P, Sada A, Robles J (2014) Listado de nombres comunes de las aves de México. Agrupación Sierra Madre. CONABIO. México. 39p.

Escalona G, Plasencia A (2012) Nuevo registro del loro frente blanca (Amazona albifrons) para la Isla de Cozumel, Quintana Roo, México. Huitzil. Revista Mexicana de Ornitología 13: 43-46.

Germosén L (1995) Hacia una farmacopea vegetal caribeña. Edición TRAMIL 7. Enda - Caribe, UAG y Universidad de Antioquia. Santo Domingo, República Dominicana. 696p.

Gómez G, Teutli C, Reyes S, Valadez R (2005) Pájaros y otras aves utilizados como animales de ornato y compañía. Revista AMMVEPE 16: 129-139. 
González K, Romero M, Escobar F, García Y (2011) Aprovechamiento de fauna silvestre por comunidades rurales en los Humedales de Catazajá - La Libertad, Chiapas, México. Universidad Autónoma Indígena de México. Ra Ximhai 7: 219-230.

Guix J, Jover L, Ruiz X (1997) Muestreos del comercio de psitácidos neotropicales en la ciudad de Barcelona, España. Ararajuba 5: 159-167.

Howell S, Webb S (2010) The birds of Mexico and Central America. Oxford University Press. EUA. 851p.

INEGI (2010) Mérida, Yucatán, México. Instituto Nacional de Estadística y Geografía. http://<www.inegi. org.mx. Fecha de consulta: 21 septiembre de 2015.

Kaufman K (2005) Guía de Campo Kaufman a las Aves Norteamericanas. Houghton Mifflin Harcourt Publishers. New York. USA. 392p.

López X, Înigo E (2009) La captura de aves silvestres en México: Una tradición milenaria y las estrategias para regularla. Biodiversitas 83: 11-15.

Monterrubio T, Villaseñor L, Marín M, López E, Fabián B, Sorani V (2007) Distribución histórica y actual del loro cabeza amarilla (Amazona oratrix) en la costa Central del Pacífico Mexicano: ventajas y limitaciones en el uso del Garp en especies bajo fuerte presión de tráfico. Ornitología Neotropical 18: 263-276.

National Geographic Society (2010) Field guide to the birds of North America. National Geographic Society. Washington, DC. EU. 480p. Pascual R, Medina E, Sandoval E, Lara E, Piña H, Martínez R, et al. (2014) Uso de reptiles entre Yoremes y Yoris en el municipio de El Fuerte, Sinaloa. Ra Ximhai 10: 195-208.

Pettingill O Jr (1970) Ornithology in the laboratory and the field. Burguess Publishing Company. Minnesota, EUA. 555p.

Ramírez J (2010) Presencia del cardenal rojo (Cardinalis cardinalis) en el suroeste de Puebla, México. Huitzil. Revista Mexicana de Ornitología 11: 42-45.

Toscano J (2006) Uso tradicional de plantas medicinales en la Vereda San Isidro, municipio de San José de Pare-Boyacá: Un estudio preliminar usando técnicas cuantitativas. Acta Biológica Colombiana 11: 137-146.

SEMARNAP (1997) Programa de conservación de la vida silvestre y diversificación productiva en el sector rural. Secretaría de Medio Ambiente, Recursos Naturales y Pesca. Instituto Nacional de Ecología. México. 207p.

SEMARNAT (2001) Guía técnica de identificación de aves canoras y de ornato autorizada por la SEMARNAT para su aprovechamiento. Dirección General de Vida Silvestre. México. 68p.

SEMARNAT (2008) Decreto por el que se adiciona un artículo 60 Bis 2 a la Ley General de Vida Silvestre. Diario Oficial de la Federación. 14 octubre 2008. México. http://dof.gob.mx/nota detalle_popup.php? codigo $=5063852$. Fecha de consulta: 21 septiembre de 2015.

SEMARNAT (2013) Tráfico ilegal de vida silvestre. Cuadernos de divulgación ambiental. Secretaria de Medio Ambiente y Recursos Naturales. México. 32p.

Sibley D (2009) The Sibley field guide to birds of Eastern North America. National Audubon Society. Alfred A. Knopf. New York. EUA. 432p. Silva R, Bernard E (2012) Wildlife sinks: Quantifying the impact of illegal bird trade in streetmarkets in Brazil. Biological Conservation 149: 16-22. 
Trejo L (2006) Aprovechamiento de las aves silvestres en Boca del Cerro municipio de Tenosique, Tabasco, México. Kuxulkab' 11: 59-63.

Uc M, Cervera M (2014) ¡Vamos a pescar!: Los niños mayas y las aves de Yucatán, México. En: VásquezDávila M (Ed.). Aves, personas y culturas. Estudios de Etno-ornitología. CONACYT/ITVO/Carteles editores/UTCH. Oaxaca, México. pp: 19-34.

UICN (2012) Red list of threatened species. Version 2012.1. Unión Internacional para la Conservación de la Naturaleza. Recuperado de http://<www.iucnredlist.org >. Fecha de consulta 3 agosto de 2015. 
